SCIENCE CHINA

Physics, Mechanics \& Astronomy

\title{
New achievements from CE-3 mission
}

\author{
YongLiao Zou, Qin Wang*, Jun Yan, YueSong Chen, and ZiYuan Ouyang \\ National Astronomical Observatories, Chinese Academy of Sciences, Beijing 100012, China
}

Received November 20, 2016; accepted February 16, 2017; published online February 22, 2017

Citation: $\quad$ Y. L. Zou, Q. Wang, J. Yan, Y. S. Chen, and Z. Y. Ouyang, New achievements from CE-3 mission, Sci. China-Phys. Mech. Astron. 60, 049631 (2017), doi: 10.1007/s11433-016-9009-6

The Chang'E-3 (CE-3) mission began with a smooth countdown and flawless launch on the Long March 3B rocket from the Xichang satellite launch center at 01:30 CST on December 2, 2013. It landed on the northeastern Imbrium basin $\left(340.49^{\circ} \mathrm{E}, 44.12^{\circ} \mathrm{N}\right)$ at $21: 11 \mathrm{CST}$ on December 14,2013 , and the Yutu rover was deployed from the lander the next morning at 04:35.

The lander was equipped with a number of remote-sensing instruments including the landing camera (LCAM), terrain camera (TCAM), extreme ultraviolet camera (EUVC), and lunar-based ultraviolet telescope (LUT). The Yutu rover successfully carried the panoramic camera (PCAM), VIS-NIR imaging spectrometer (VNIS), active particle-induced X-ray spectrometer (APXS), and lunar penetrating radar (LPR) [1-3].

Three cameras (LCAM, TCAM, and PCAM) investigated the morphological features and geological structures near the landing area. The EUVC monitored activities in the Earth's plasmasphere at extreme ultraviolet wavelengths (measurement band center frequency $30.4 \pm 0.5 \mathrm{~nm}$, bandwidth $\leq 5 \mathrm{~nm}$ ). The LUT performed astronomical observations at near-ultraviolet wavelengths $(245-340 \mathrm{~nm})$. The VNIS (visible-nearinfrared 450-950 nm, short-wave infrared 900-2400 nm) and APXS detected the mineralogical and chemical compositions along the traverse path. The LPR obtained pioneering measurements of the lunar subsurface.

After the lander released the Yutu rover, the eight science instruments began operating. The Yutu rover started traversing along a planned path covering a distance of $114 \mathrm{~m}$.

*Corresponding author (email: wangqin@bao.ac.cn)
To analyze the topography, landform, and geology of the area surrounding the landing site (Sinus Iridum and $45 \mathrm{~km} \times$ $70 \mathrm{~km}$ of the landing area), high-resolution topography data, image data, and geological data were obtained [4].

Thus far, eight science instrument calibration procedures, data processing methods, and inversion models have been completed [5-12]. The total science data size was $6.69 \mathrm{~TB}$ as of April 4, 2016.

The LPR detection and integrated TCAM \& PCAM data interpretation have identified more than seven subsurface layers, suggesting that the landing region has experienced complex geological processes such as basalts and pyroclastic have filled in the Imbrium basin since the Imbrian (3.9-3.2 Ga) and Eratosthenian (3.2-1.5 Ga) epochs [3]. The LPR and PCAM data provides a higher resolution to estimate lunar regolith parameters such as permittivity, density, conductivity, and $\mathrm{FeO}+\mathrm{TiO}_{2}$ content. The surface regolith thickness of the CE-3 landing area is $(1.24 \pm 0.10) \mathrm{m}$. Meteorite impacts might have been a vital influence to the thickness and growth rate of the regolith [13].

The LPR consists of two types of antenna with two channels: 60 (Channel 1) and $500 \mathrm{MHz}$ (Channel 2). The LPR observations at $500 \mathrm{MHz}$ reveal four major stratigraphic zones from the surface to a depth of $20 \mathrm{~m}$ : a layered reworked zone $(<1 \mathrm{~m})$, ejecta layer $(\sim 2-6 \mathrm{~m})$, paleoregolith layer $(\sim 4-11 \mathrm{~m})$, and the underlying mare basalts. In addition, the LPR measurements indicate a mean of about 5-10 m/Gyr for the surface regolith, which is at least 4-8 times larger than the previous estimation [14]. These results indicate that the landing site features thin regolith and geological evolution history.

The LPR measurements also detected three underlying 
basalt units at depths of 195,215 , and $345 \mathrm{~m}$ at the CE-3 landing area, and indicated significant volcanic eruptions in the Imbrium basin until $2.5 \mathrm{Ga}$, which is different from the gradual cessation of magma eruption in the Imbrium basin [15].

Correlated compositional and mineralogical investigations of the lunar soil at the landing site were determined by the Yutu rover with in situ measurements by APXS and VNIS. The CE-3 regolith was derived from olivine-normative basaltic rocks with higher $\mathrm{FeO}(\sim 22.8 \mathrm{wt} \%)$ and $\mathrm{TiO}_{2}$ $(\sim 5.0 \mathrm{wt} \%)$ [16]. Furthermore, the basalt has a high $\mathrm{Al}_{2} \mathrm{O}_{3}$ content $(\sim 11 \mathrm{wt} \%)$ [17], these results indicate a new type of mare basalt.

The PCAM captured the first in situ lunar opposition effect $(\mathrm{OE})$. The in situ phase curve covers a large phase range from $2^{\circ}$ to $141^{\circ}$. The photometric inversions results reveal that submicroscopic dust is present in the landing areas, and both the coherent backscattering and shadow hiding are responsible for the strong OE. The grains at the landing site are brighter, more transparent, and appear to be better crystallized than the average maria basaltic grains. The results indicate that the phase-reddening effect appears to be present in the in situ phase curves because of the strong mineral absorption features [18].

Based on more than 1300 images from the EUVC, a global and instantaneous meridian view (side view) of the Earth's plasmasphere is obtained. During the observation period, the results indicated that the plasmasphere had three bulges that coincided with the expansion phase of three substorms. This implies a causal relationship between the substorms and the formation of the three bulges on the plasmasphere. The measurements suggest that there is a strong negative correlation between the plasmaspheric configuration and geomagnetic activity intensity [19].

This was the first time the LUT on the lander performed observations of the diurnal sky at ultraviolet wavelengths. The observed region covers about 2400 square degrees, including low-galactic-latitudes $b<15^{\circ}$. The LUT additionally performed low-galactic-latitude sky surveys as a complement to the NASA GALEX (galaxy evolution explorer) mission [20].

GQ Dra is a short-period eclipsing binary in a double stellar system that was discovered by Hipparcos. The photometric solutions are determined using the Wilson-Devinney (W-D) method. It discovered that GQ Dra is a classical Algol-type semi-detached binary where the secondary component is filling the critical Roche lobe. The semi-detached configuration of GQ Dra indicates that a mass transfer from the less massive to more massive component should take place. All results reveal that GQ Dra is a mass-transferring semi-detached binary in a double system that was formed from an initially detached binary star [21].

Using the W-D method, it is discovered that the neglected eclipsing binary Algol V548 Cygni presented three additional stellar companions. This is in agreement with the presence of a large quantity of a third source of light in the system. The masses of these companions are estimated as $m \sin i \sim 1.09 M_{\mathrm{A}}$, $0.20 M_{\mathrm{A}}$, and $0.52 M_{\mathrm{A}}$. They are orbiting the central binary with orbital periods of about 5.5, 23.3, and 69.9 years, i.e., in a 1:4:12 resonance orbit. Their orbital separations are about 4.5, 13.2, and 26.4 AU, respectively. No clear long-term changes in orbital period were observed, indicating that the contributions of the mass transfer and mass loss due to magnetic braking to the period variations are comparable. The detection of three possible additional stellar components orbiting a typical Algol in a multiple system makes V548 Cygni a very interesting binary [22].

Besides the $\mathrm{OH}$ resonant emission at $3087 \AA$, the LUT bandpass covers two strong resonant emission lines $\mathrm{MgI}$ (22853) and AlI (23092). It enables us to study the $\mathrm{OH}$ content from the diffuse background level recorded in the images acquired by LUT. An unprecedented upper limit of $\mathrm{OH}$ content in the lunar exosphere was obtained from the in situ measurements taken by LUT. The inferred column density and surface concentration are $<10^{11} \mathrm{~cm}^{-2}$ and $<10^{4}$ $\mathrm{cm}^{-3}$ for the $\mathrm{OH}$ radicals, respectively, which is close to the value predicted by the sputtering model. The upper limit of $<10^{4} \mathrm{~cm}^{-3}$ derived for the $\mathrm{OH}$ radicals is lower than that derived from the HST low-resolution spectroscopy by about two orders of magnitude, and is lower than that inferred from the mass spectra taken by the Chandrayaan-1 mission by about 6 orders of magnitude [23].

The CE-5 mission, scheduled for 2017, will be China's first sample-return mission, aiming to return at least $2 \mathrm{~kg}$ of lunar soil and rock samples.

The Chang'E-4 mission, phase two of the China lunar exploration program, represents China's first attempt to explore the far side of the moon. The CE-4 mission includes a lander, rover, and telecommunications relay, currently scheduled to launch in 2018 .

The scientific objectives of future lunar exploration missions are space and astronomy, geology and structure, and resources and environment. Proposed future lunar missions include robotic lunar missions and human lunar exploration. China is currently working on robotic missions to conduct scientific research and support future human surface missions. Robotic science missions would detect the lunar south/north pole and return samples. Thus, the robotic missions will continue well into the next decade to meet high-priority science objectives and prepare for future human missions to the moon. Human lunar exploration would include human orbiter lunar exploration, a manned lunar landing, and a manned lunar scientific station.

This work was supported by the Major Program of the National Natural Science Foundation of China (Grant No. 41590851).

1 W. H. Ip, J. Yan, C. L. Li, and Z. Y. Ouyang, Res. Astron. Astrophys. 
14, 1511 (2014).

2 C. Li, J. Liu, X. Ren, W. Zuo, X. Tan, W. Wen, H. Li, L. Mu, Y. Su, H. Zhang, J. Yan, and Z. Ouyang, Space Sci Rev 190, 85 (2015).

3 L. Xiao, P. Zhu, G. Fang, Z. Xiao, Y. Zou, J. Zhao, N. Zhao, Y. Yuan, L. Qiao, X. Zhang, H. Zhang, J. Wang, J. Huang, Q. Huang, Q. He, B. Zhou, Y. Ji, Q. Zhang, S. Shen, Y. Li, and Y. Gao, Science 347, 1226 (2015).

4 C. L. Li, L. L. Mu, X. D. Zou, J. J. Liu, X. Ren, X. G. Zeng, Y. M. Yang, Z. B. Zhang, Y. X. Liu, W. Zuo, and H. Li, Res. Astron. Astrophys. 14, 1514 (2014).

5 X. Tan, J. J. Liu, C. L. Li, J. Q. Feng, X. Ren, F. F. Wang, W. Yan, W. Zuo, X. Q. Wang, and Z. B. Zhang, Res. Astron. Astrophys. 14, 1682 (2014).

6 Z. P. He, B. Y. Wang, G. Lü, C. L. Li, L. Y. Yuan, R. Xu, B. Liu, K. Chen, and J. Y. Wang, Res. Astron. Astrophys. 14, 1567 (2014).

7 B. Liu, C. L. Li, G. L. Zhang, R. Xu, J. J. Liu, X. Ren, X. Tan, X. X. Zhang, W. Zuo, and W. B. Wen, Res. Astron. Astrophys. 14, 1578 (2014).

8 G. Y. Fang, B. Zhou, Y. C. Ji, Q. Y. Zhang, S. X. Shen, Y. X. Li, H. F. Guan, C. J. Tang, Y. Z. Gao, W. Lu, S. B. Ye, H. D. Han, J. Zheng, and S. Z. Wang, Res. Astron. Astrophys. 14, 1607 (2014).

9 H. B. Zhang, L. Zheng, Y. Su, G. Y. Fang, B. Zhou, J. Q. Feng, S. G. Xing, S. Dai, J. D. Li, Y. C. Ji, Y. Z. Gao, Y. Xiao, and C. L. Li, Res. Astron. Astrophys. 14, 1633 (2014).

10 S. Dai, Y. Su, Y. Xiao, J. Q. Feng, S. G. Xing, and C. Y. Ding, Res. Astron. Astrophys. 14, 1642 (2014).

11 Y. Su, G. Y. Fang, J. Q. Feng, S. G. Xing, Y. C. Ji, B. Zhou, Y. Z. Gao, H. Li, S. Dai, Y. Xiao, and C. L. Li, Res. Astron. Astrophys. 14, 1623 (2014).

12 X. H. Fu, C. L. Li, G. L. Zhang, Y. L. Zou, J. J. Liu, X. Ren, X. Tan, X.
X. Zhang, W. Zuo, W. B. Wen, W. X. Peng, X. Z. Cui, C. M. Zhang, and H. Y. Wang, Res. Astron. Astrophys. 14, 1595 (2014).

13 Z. Dong, G. Fang, Y. Ji, Y. Gao, C. Wu, and X. Zhang, Icarus 282, 40 (2017).

14 W. Fa, M. H. Zhu, T. Liu, and J. B. Plescia, Geophys. Res. Lett. 42, 10,179 (2015)

15 J. Zhang, W. Yang, S. Hu, Y. Lin, G. Fang, C. Li, W. Peng, S. Zhu, Z. He, B. Zhou, H. Lin, J. Yang, E. Liu, Y. Xu, J. Wang, Z. Yao, Y. Zou, J. Yan, and Z. Ouyang, Proc. Natl. Acad. Sci. 112, 5342 (2015).

16 Z. Ling, B. L. Jolliff, A. Wang, C. Li, J. Liu, J. Zhang, B. Li, L. Sun, J. Chen, L. Xiao, J. Liu, X. Ren, W. Peng, H. Wang, X. Cui, Z. He, and J. Wang, Nat. Commun. 6, 8880 (2015).

17 H. Zhang, Y. Yang, Y. Yuan, W. Jin, P. G. Lucey, M. H. Zhu, V. G. Kaydash, Y. G. Shkuratov, K. Di, W. Wan, B. Xu, L. Xiao, Z. Wang, and B. Xue, Geophys. Res. Lett. 42, 6945 (2015).

18 W. Jin, H. Zhang, Y. Yuan, Y. Yang, Y. G. Shkuratov, P. G. Lucey, V. G. Kaydash, M. H. Zhu, B. Xue, K. Di, B. Xu, W. Wan, L. Xiao, and Z. Wang, Geophys. Res. Lett. 42, 8312 (2015).

19 H. He, C. Shen, H. Wang, X. Zhang, B. Chen, J. Yan, Y. Zou, A. M. Jorgensen, F. He, Y. Yan, X. Zhu, Y. Huang, and R. Xu, Sci. Rep. 6, 32362 (2016).

20 J. Wang, J. S. Deng, J. Cui, L. Cao, Y. L. Qiu, and J. Y. Wei, Adv. Space Res. 48, 1927 (2011), arXiv: 1108.0727.

21 S. B. Qian, X. Zhou, L. Y. Zhu, M. Zejda, B. Soonthornthum, E. G. Zhao, J. Zhang, B. Zhang, and W. P. Liao, Astron. J. 150, 193 (2015).

22 L. Y. Zhu, X. Zhou, J. Y. Hu, S. B. Qian, L. J. Li, W. P. Liao, X. M. Tian, and Z. H. Wang, Astron. J. 151, 107 (2016).

23 J. Wang, C. Wu, Y. L. Qiu, X. M. Meng, H. B. Cai, L. Cao, J. S. Deng, X. H. Han, and J. Y. Wei, Planet. Space Sci. 109-110, 123 (2015), arXiv: 1502.04287 . 